\title{
Counting and cutting cycles of lines and rods in space*
}

\author{
Bernard Chazelle \\ Dept. Comput. Sci., Princeton Univ., Princeton, NJ 08544, USA
}

\section{Herbert Edelsbrunner}

Dept. Comput. Sci., Univ. Illinois, Urbana, IL 61801, USA

\section{Leonidas J. Guibas}

Lab. Comput. Sci., MIT, Cambridge, MA 02139 and DEC SRC, Palo Alto, CA 94301, USA

\section{Richard Pollack}

Courant Inst. Math. Sci., New York Univ., New York, NY 10012, USA

\section{Raimund Seidel}

Dept. Comput. Sci. Electr. Engin., Univ. California, Berkeley, CA 94720, USA

\section{Micha Sharir}

Courant Inst. Math. Sci., New York Univ., New York, NY 10012, USA

School Math. Sci., Tel Aviv Univ., Tel Aviv 69978, Israel

\section{Jack Snoeyink}

Dept. Comput. Sci., Stanford Univ., Stanford, CA 94305, USA

Communicated by Kurt Mehlhorn

Submitted 2 October 1990

Accepted 1 February 1991

\footnotetext{
* Bernard Chazelle wishes to acknowledge the National Science Foundation for supporting this research in part under Grant CCR-9002352. Herbert Edelsbrunner acknowledges the support of the National Science Foundation under grants CCR-8714565 and CCR-8921421. Richard Pollack was supported in part by NSF grant CCR-8901484, NSA grant MDA904-89-H-2030, and DIMACS, a Science and Technology Center under NSF grant STC88-09648. Raimund Seidel acknowledges support by NSF grant CCR-8809040. Mich Sharir was partially supported by the Office of Naval Research under Grant N00014-87-K-0129, by the National Science Foundation under Grant CCR-89-01484, and by grants from the U.S.-Israeli Binational Science Foundation and the Fund for Basic Research administered by the Israeli Academy of Sciences.
} 


\begin{abstract}
Chazelle, B., H. Edelsbrunner, L.J. Guibas, R. Pollack, R. Seidel, M. Sharir and J. Snoeyink, Counting and cutting cycles of lines and rods in space, Computational Geometry: Theory and Applications 1 (1992) 305-323.

A number of rendering algorithms in computer graphics sort three-dimensional objects by depth and assume that there is no cycle that makes the sorting impossible. One way to resolve the problem caused by cycles is to cut the objects into smaller pieces. In this paper we address the problem of estimating how many such cuts are always sufficient. We also consider a few related algorithmic and combinatorial geometry problems. For example, we demonstrate that $n$ lines in space can be sorted in randomized expected time $\mathrm{O}\left(n^{1 / 3+c}\right)$, provided that they define no cycle. We also prove an $O\left(n^{7 / 4}\right)$ upper bound on the number of points in space so that there are $n$ lines with the property that for each point there are at least three noncoplanar lines that contain it.
\end{abstract}

Keywords. Discrete and computational geometry; hidden surface removal; depth sort; lines; line segments; cycles; incidences.

\title{
1. Introduction
}

The hidden surface removal problem in computer graphics is the problem of computing the view of a scene from some viewpoint, see e.g. [7]. We are interested in the case where the scene is given as a collection of pairwise disjoint polyhedral objects. Since only the boundaries of the objects are relevant we may as well assume that the scene is given by a collection of two-dimensional polygons with pairwise disjoint relative interiors.

Many of the popular algorithms that work in object space (as opposed to image space) are based on an ordering of the polygons by depth, that is, if $P$ and $Q$ are two polygons and $P$ obstructs (parts of) $Q$ as seen from the viewpoint, then $P$ precedes $Q$ in this order. Notable examples are the painter's algorithm [7], which 'paints' the polygons according to this order from back to front, and the output-sensitive methods of Overmars and Sharir [11], which process the polygons from front to back. These algorithms are correct only if an ordering of the polygons as described exists, that is, if there is no sequence of polygons $P_{1}, P_{2}, \ldots, P_{k}$ so that $P_{i}$ obstructs (parts of) $P_{i+1}$ for $1 \leqslant i<k$ and $P_{k}$ obstructs (parts of) $P_{1}$.

Of course, if such a cycle of polygons occurs, we can decompose (cut) one polygon into smaller pices and thus eliminate the cycle. Such a method has been proposed by Fuchs, Kedem and Naylor [8]; the three-dimensional version of their method recursively decomposes the polygons using planes and stores the planes in the nodes of a binary tree. The construction of a view, as well as an 'order of obstructions', from a given viewpoint reduces to a traversal of this tree. The decomposition effectively removes all cycles for all possible viewpoints, but depending on the choice of the planes it can require a rather large amount of storage. More recently, Paterson and Yao [13] showed how to choose the planes 
so that the storage does not exceed $\mathrm{O}\left(n^{2}\right)$, where $n$ is the total number of edges of the polygons, and they also demonstrate that this bound is tight for the three-dimensional version of the recursive partitioning method of [8].

In this paper we consider algorithmic and combinatorial questions related to sorting objects by depth and cutting them to eliminate cycles. Our approach differs from the above as we consider a fixed viewpoint, which we assume to be at $(0,0,+\infty)$. We further simplify the problems by considering lines and rods in place of polygons. Even though the resulting questions retain the inherent difficulty of the polygon problems they are easier to discuss and are more suitable for demonstrating the methods and ideas.

This paper should be viewed as part of a broader effort to understand the algorithmic and combinatorial aspects of problems for lines and rods in space, see $[3,4]$. The interaction of lines in space, or more generally of one-dimensional objects in three-dimensional space, is the least understood subproblem that comes up in the study of polyhedral three-dimensional objects. Much easier in many ways are planes (two-dimensional objects) and points (zero-dimensional objects), in part because they can be mapped into each other by duality, see e.g. [6]. Our study is also related to recent investigations of finite sets of lines and of weaving patterns in $[12,17,18]$. In particular, we will repeatedly make use of the results on the unrealizahility of certain weaving patterns of [12].

This paper is organized as follows. Section 2 considers the problem of sorting lines and/or rods relative to a viewpoint at infinity. Section 3 proves lower and upper bounds on the maximum number of cycles in a realizable bipartite weaving, and Section 4 uses these results to derive a subquadratic bound on the number of cuts necessary to eliminate all cycles. Section 5 studies an incidence problem for lines and points in space, and Section 6 discusses the maximum number of cycles in realizable weavings that are not necessarily bipartite. Section 7 formulates several problems that remain open.

\section{Sorting rods and lines in space}

Let $R$ be a set of $n$ pairwise disjoint rods in space, where a rod is a relatively open connected subset of a line. We consider the problem of sorting the rods along a given direction. Assume this is the vertical direction, given by the $z$-axis, and that no rod in $R$ is vertical. For two rods $a, b \in R$ we say that $a$ is below $b$, for short $a<b$, if there is a vertical line $l$ so that $a \cap l \neq \emptyset, b \cap l \neq \emptyset$, and the $z$-coordinate of $a \cap l$ is smaller than that of $b \cap l$. With this notation sorting $R$ means finding an ordering $r_{1}, r_{2}, \ldots, r_{n}$ of the rods so that $r_{i}$ is not above $r_{j}$ if $i<j$. In other words, $r_{1}, r_{2}, \ldots, r_{n}$ is a linear extension of the relation $(R,<)$. Of course, such a linear extension exists only if $(R,<)$ is a partial order.

We can now formally define what we require from an algorithm that sorts $R$. If $(R,<)$ is a partial order the algorithm should output a linear extension of $(R,<)$. 

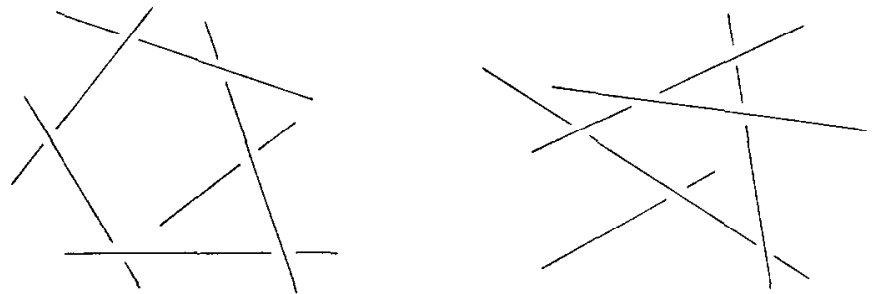

Fig. 1. We show the view from point $(0,0,+\infty)$ of two sets of rods in space. The left set contains a cycle whereas the right set is cycle-free.

In this case, the rods can be taken vertically to infinity, one by one in a non-interfering manner, like in the game of pick-up-sticks. Otherwise, we want the algorithm to output a cycle, that is, a sequence of rods $r_{1}, r_{2}, \ldots, r_{j}$ with $r_{1}<r_{2}<\cdots<r_{j}<r_{1}$. Fig. 1 shows an example for each case.

There is a straightforward algorithm that runs in time $\mathrm{O}(n \log n+k)$ in the worst case, where $k$ is the number of pairs of rods that are related by $<$. No faster algorithm is known at this time.

1. Construct the directed graph $\mathscr{G}=(R, A)$ with $(a, b) \in A$ iff $a<b$.

2. Run a topological sorting algorithm on $\mathscr{G}$; it will either compute a linear extension of $(R,<)$ or detect a cycle in the graph.

Note that $a$ and $b$ are related iff the vertical projections of $a$ and $b$ onto the $x y$-plane intersect. Step 1 can thus be completed in time $\mathrm{O}(n \log n+k)$ using the optimal line segment intersection algorithm of Chazelle and Edelsbrunner [2] for the vertical projections of the rods. Using $\mathscr{G}$, standard algorithms can be used to compute a linear extension of $(R,<)$ in time proportional to the size of $\mathscr{G}$, that is, $\mathrm{O}(n+k)\lceil 10\rceil$.

There are several questions raised by this straightforward result, some of which will be addressed in this paper. One is whether a set of $n$ rods in space can be sorted in subquadratic time, even if $k=\Theta\left(n^{2}\right)$. An affirmative answer for the special case where each rod is a line is given later in this section. Another question concerns the elimination of cycles. A cycle in $(R,<)$ can be eliminated by cutting any one of its rods at an appropriate point. What is the maximum number of cuts necessary to remove all cycles, where the maximum is taken over all sets of $n$ rods? Bounds on this number will be given in Sections 4 and 6 .

We now consider the sorting problem for the special case where $R=L$ is a set of $n$ lines in space. We assume that the lines are pairwise disjoint and that their vertical projections onto the $x y$-plane are pairwise non-parallel. These assumptions are crucial for our considerations and cannot be dropped easily. The assumptions imply that $\mathscr{G}=(L, A)$ is a tournament (that is, a directed graph so that between every two nodes there is exactly one arc) which means that it 
contains a cycle of length three if it contains a cycle at all. If $\mathscr{G}$ has no cycle then $(L,<)$ is a total order, which implies that $L$ can be sorted in time $\mathrm{O}(n \log n)$ by any standard comparison-based algorithm [9]. If $(L,<)$ has cycles then there are two possibilities. Either the sorting algorithm runs into an inconsistency or the cycles go unnoticed. For most sorting algorithms inconsistencies are not a problem, that is, they still finish in about the same amount of time as for a cycle-free input-only that the result is not sorted. The algorithm for which this is most easily seen is merge-sort: a recursive call returns a sequence of lines so that any two adjacent lines are in the correct order, and this property is maintained when two sequences are merged.

The problem that remains is to check whether a given sequence of lines, $l_{1}, l_{2}, \ldots, l_{n}$, is really sorted. Here we use the following result that can be found in [4]. Given $m_{1}$ blue and $m_{2}$ red lines in space and any real number $\varepsilon>0$, there is an algorithm that tests whether all blue lines lie below all red lines in randomized expected time $\mathrm{O}\left(\left(m_{1}+m_{2}\right)^{4 / 3+\varepsilon}\right)$. To test $l_{1}, l_{2}, \ldots, l_{n}$ we apply this algorithm after coloring the first half blue and the second half red and then recurse for both halves. The amount of time required obeys the recurrence relation $T(n)=2 T(n / 2)+\mathrm{O}\left(n^{4 / 3+\varepsilon}\right)$ which solves to $\mathrm{O}\left(n^{4 / 3+\varepsilon}\right)$. We summarize the results of this section.

Theorem 2.1. (i) The sorting problem for $n$ pairwise disjoint rods in space can be solved in worst-case time $\mathrm{O}(n \log n+k)$, where $k$ is the number of pairs related by $\prec$.

(ii) The sorting problem for $n$ lines in space, pairwise disjoint and no two with parallel vertical projections, can be solved in randomized expected time $\mathrm{O}\left(n^{4 / 3+\varepsilon}\right)$.

Remark. Part (i) of Theorem 2.1 suggests that we look for a sorting algorithm for rods that runs in subquadratic time even when $k$ is proportional to $n^{2}$. Preliminary positive results have been obtained and will possibly be reported elsewhere. For example, a subquadratic algorithm can be found for the bipartite case of the problem. In this case $R$ can be partitioned into sets $H$ and $V$ so that in the vertical projection onto the $x y$-plane every rod in $H$ intersects every rod in $V$, but no two rods in $H$ intersect and neither do any two rods in $V$. Here are the main ideas for this result.

Run a sorting algorithm, sequential or parallel, on $R$. Any rod in $H$ can be compared in constant time with any rod in $V$. However, when two rods in $H$ are compared, then we need to consult the rods in $V$ to decide whether they form a cycle and, if not, in which order we should arrange them. This can be done in time $\mathrm{O}\left(n^{\alpha}\right)$, for $\alpha<1$, using a suitable data structure for $V$ based on the results in [4]. In case the rods in $V$ do not force any one order then we just order the two rods in $H$ according to preassigned indices. The same can be said with the roles of $H$ and $V$ interchanged. Of course, many details have to be filled in, most 
prominently an argument that the fairly arbitrary decision in the last case does not lead to inconsistences. Finally, when an ordering is computed we check whether it is acyclic using results of $[3,4]$.

\section{Counting cycles in the bipartite case}

In this section we study the number of cycles in what we call realizable bipartite weavings to be defined shortly-realizable weavings that are not necessarily bipartite will be discussed in Section 6. To avoid confusion in the following discussion, which is partly planar and partly spatial, we call the projection of a rod a line segment and use primes to indicate projection.

A weaving is a pair $\left(R^{\prime},<\right)$ where $R^{\prime}$ is a planar arrangement of lines of (relatively open) line segments and $<$ is an antisymmetric, non-reflexive binary relation on $R^{\prime}$ which is defined precisely on those pairs in $R^{\prime}$ that intersect. Thus, $\left(R^{\prime},<\right)$ can be thought of as a directed version of the intersection graph of $R^{\prime}$. The weaving $\left(R^{\prime},<\right)$ is realizable if there is a collection of rods $R$ in space for which $R^{\prime}$ is the set of orthogonal projections onto the $x y$-plane of the rods in $R$ and $r^{\prime}<s^{\prime}$ iff the rod $r$ is below $s$. We say that $R$ realizes $\left(R^{\prime},<\right)$. Finally, we say that a weaving $\mathscr{W}=\left(R^{\prime},<\right)$ is an $m$-by- $n$ bipartite weaving if $R^{\prime}$ can be partitioned into two sets $H^{\prime}=\left\{h_{1}^{\prime}, h_{2}^{\prime}, \ldots, h_{m}^{\prime}\right\}$ (which we think of as a collection of $m$ more or less horizontal line segments) and $V^{\prime}=\left\{v_{1}^{\prime}, v_{2}^{\prime}, \ldots, v_{n}^{\prime}\right\}$ (which we think of as a collection of $n$ more or less vertical line segments) such that each line segment in $H^{\prime}$ meets the line segments in $V^{\prime}$ in the order $v_{1}^{\prime}, v_{2}^{\prime}, \ldots, v_{n}^{\prime}$ and each line segment in $V^{\prime}$ meets the line segments in $H^{\prime}$ in the order $h_{1}^{\prime}, h_{2}^{\prime}, \ldots, h_{m}^{\prime}$. Moreover, the line segments in $H^{\prime}$ are pairwise disjoint as are the line segments in $V^{\prime}$.

Note that any bipartite weaving forms a checkerboard pattern as illustrated in Fig. 2, which shows two bipartite weavings. If a bipartite weaving $\left(R^{\prime},<\right)$ is realizable then the partition of $R^{\prime}$ into $H^{\prime}$ and $V^{\prime}$ corresponds to a partition of a realizing set $R$ into sets $H$ and $V$. By reference to the orderings of the line segments defined in a bipartite weaving we call two rods $h_{i}, h_{j} \in H\left(\right.$ and $v_{i}, v_{j} \in V$ ) adjacent if $j=i+1$.
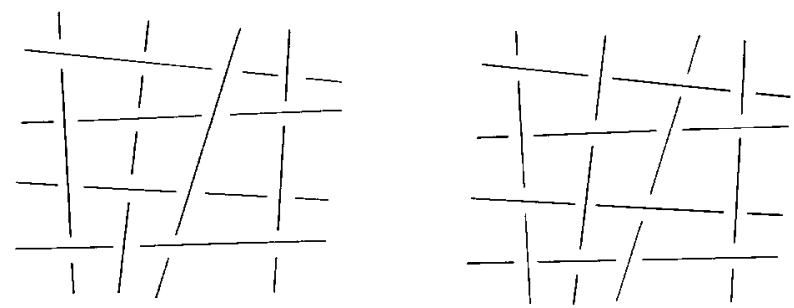

Fig. 2. Both weavings are bipartite but only the one to the left is realizable. 
Not all bipartite weavings are realizable. For example, any 4-by-4 bipartite weaving where each rod strictly alternates between crossing 'over' and 'under' (Fig. 2 right) is nonrealizable as shown by Pach, Pollack and Welzl [12]. Such weavings are called perfect 4-by-4 weavings. We will make use of this nonrealizability result when we study cycles in realizable bipartite weavings.

The notation used in this section is as follows. $\mathscr{W}$ is a realizable bipartite weaving defined by a set $H \cup V$ of $2 n$ rods in space, with $|H|=|V|=n$. (The extension of our results to the case where $|H| \neq|V|$ is straightforward.) $\mathscr{G}=(H \cup V, A)$ is the (bipartite) digraph that corresponds to $\mathscr{W}$, that is, $(a, b) \in A$ iff $a<b$ for $a, b \in I U \cup V$. This section studies the maximum number of cycles of some type, where the maximum is taken over all realizable bipartite weavings $\mathscr{W}$ of size $n$-by- $n$. A cycle in $\mathscr{W}$ (or, equivalently, in $\mathscr{G}$ ) has length at least 4 . Every cycle of $\mathscr{G}$ of length more than 4 contains a shorter cycle and therefore also a cycle of length 4; we call a cycle of length 4 a tetragonal cycle. The maximum number of tetragonal cycles is $\Theta\left(n^{4}\right)$; the upper bound is trivial and a lower bound construction is indicated in Fig. 3 .

A much more interesting problem than counting tetragonal cycles is to count the number of so-called elementary cycles. An elementary cycle is a tetragonal cycle defined by two adjacent rods in $H$ and two adjacent rods in $V$. We define $f(n)$ as the maximum number of elementary cycles in a realizable bipartite weaving of size $n$-by- $n$. Note that if we cut a rod at some point we remove at most four elementary cycles. Hence, $f(n) / 4$ is a lower bound on the maximum number of cuts necessary to remove all cycles in a realizable $n$-by- $n$ weaving. We now state and prove the main result of this section.

Theorem 3.1. There are positive constants $c_{1}$ and $c_{2}$ so that $c_{1} n^{4 / 3} \leqslant f(n) \leqslant c_{2} n^{3 / 2}$.

Proof. We first prove the upper bound and then the lower bound. The argument for the upper bound uses forbidden subgraphs and the nonrealizability of the perfect 4-by-4 weaving mentioned above. The specific results on forbidden subgraphs that we use is that any bipartite graph with $k$ plus $l$ nodes cannot have more than $c\left(k l^{1 / 2}+l\right)$ arcs, for some positive constant $c$, if it contains no $K_{2,2}$ (see e.g. [1, chapter VI]).

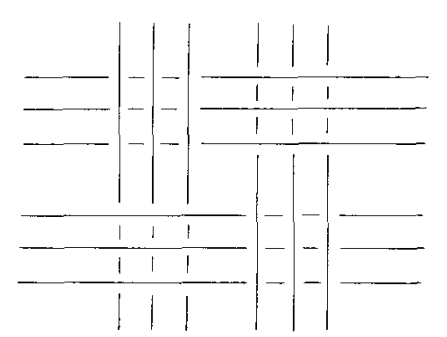

Fig. 3. This bipartite weaving is obviously realizable and has about $(n / 4)^{4}$ tetragonal cycles. 


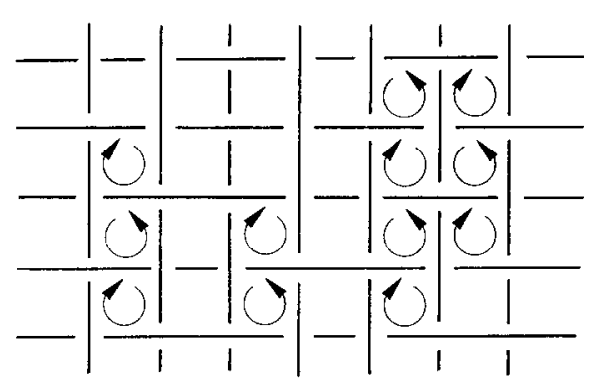

Fig. 4. A 5-by-7 weaving with $\mathrm{cw}$ and $\mathrm{ccw}$ elementary cycles marked. It is not realizable because $h_{2}$, $h_{3}, h_{4}, h_{5}, v_{1}, v_{2}, v_{5}, v_{6}$ form a perfect 4-by-4 weaving.

Let $\mathscr{W}$ be a realizable $n$-by- $n$ weaving defined by sets of rods $H=$ $\left\{h_{1}, h_{2}, \ldots, h_{n}\right\}$ and $V=\left\{v_{1}, v_{2}, \ldots, v_{n}\right\}$. In the figures, we draw the rods of $H$ about horizontally in scquence from top to bottom and the rods of $V$ about vertically in sequence from left to right (see Fig. 4). We present the argument only for counterclockwise (ccw) elementary cycles; symmetry implies the same bound for clockwise (cw) elementary cycles.

Think of $\mathscr{W}$ of an $(n-1)$-by- $(n-1)$ matrix with an entry equal to 1 where $\mathscr{W}$ has a ccw elementary cycle and 0 everywhere else. Formally, we define $\mathscr{A}=\left(a_{i j}\right)$ with

$$
a_{i j}= \begin{cases}1 & \text { if } h_{i}<v_{j}<h_{i+1}<v_{j+1}<h_{i}, \text { and } \\ 0 & \text { otherwise }\end{cases}
$$

Note that if $a_{i j}=1$ then all adjacent entries (to the left, to the right, above, and below) are equal to 0 , even if $\mathscr{W}$ is not realizable. It follows that if there are indices $1 \leqslant i_{1}<i_{2} \leqslant n-1$ and $1 \leqslant j_{1}<j_{2} \leqslant n-1$ with $a_{i_{1} j_{1}}=a_{i_{1} j_{2}}=a_{i_{2} j_{1}}=a_{i_{2} j_{2}}=1$ then we necessarily have $i_{1} \leqslant i_{2}-2$ and $j_{1} \leqslant j_{2}-2$. Thus, no two of the 8 rods $h_{i_{1}}$, $h_{i_{1}+1}, h_{i_{2}}, h_{i_{2}+1}, v_{i_{1}}, v_{j_{1}+1}, v_{j}, v_{i_{2}+1}$ are the same, and together they form a perfect 4 -by-4 weaving. It follows that $\mathscr{A}$ cannot have such a 2 -by-2 minor if $\mathscr{W}$ is realizable. Now think of $\mathscr{A}$ as the adjacency matrix of a bipartite graph with $n-1$ and $n-1$ nodes. Since $\mathscr{A}$ contains no $2-$ by- 2 minor with four 1 's this graph contains no $K_{2,2}$. By the forbidden subgraph result mentioned earlier it follows that there are at most $c\left(n^{3 / 2}+n\right)$ arcs. The upper bound of Theorem 3.1 follows with $c_{2}=4 c$ because each $\mathrm{ccw}$ elementary cycle is represented by an arc in this graph, and because the same bound holds for the cw elementary cycles.

To prove the lower bound we construct a set $H \cup V$ of rods that defines a bipartite weaving with $\Omega\left(n^{4 / 3}\right)$ elementary cycles. It is based on a configuration of $k$ points and $l$ lines in the plane that realize $\Omega\left(k^{2 / 3} l^{2 / 3}+k+l\right)$ incidences, where an incidence is a point-line pair so that the point lies on the line. A detailed description of such a configuration can be found in [6]. We remark that this number of incidences is asymptotically optimal as shown in [16,5]. For the construction we assume that $n$ is even and we let $P$ be a set of $n / 2$ points and $L$ 
be a set of $n / 2$ lines in the $y z$-plane realizing $\Omega\left(n^{4 / 3}\right)$ incidences. Without loss of generality we assume that no line in $L$ is vertical and that no two points in $P$ lie on a common vertical line. From $P$ we construct a set $V_{1}$ that contains for each $p \in P$ the line $v_{p}$ parallel to the $x$-axis through point $p$. From $L$ we construct a set $H_{1}$ that contains for each $l \in L$ the line $h_{l}$ that is the orthogonal projection of $l$ onto the plane $x=\sigma_{l}$, with $\sigma_{l}$ the slope of $l$ within the $y z$-plane. Clearly, $h_{l} \cap v_{p} \neq \emptyset$ iff $p \in l$, so we have $\Omega\left(n^{4 / 3}\right)$ pairs of intersecting lines.

We next replace each line by two nearby lines so that each intersection becomes a cycle. Choose $\delta>0$ and for each line $v_{p}$ define two lines, $v_{p}^{-}$and $v_{p}^{+}$, as follows. If $\rho=\left(0, \pi_{2}, \pi_{3}\right)$ then $v_{p}^{-}$intersects the $y z$-plane in point $\left(0, \pi_{2}-\right.$ $\left.\delta, \pi_{3}\right)$ and $v_{p}^{+}$intersects the $y z$-plane in point $\left(0, \pi_{2}+\delta, \pi_{3}\right)$. Furthermore, $v_{p}^{-}$ lies in the plane $y=\pi_{z}-\delta$ and has slope $-\delta$ within this plane. Similarly, $v_{p}^{+}$lies in $y=\pi_{2}+\delta$ and has slope $+\delta$, see Fig. 5. By construction, $h_{l}$ intersects $v_{p}^{-}$and $v_{p}^{+}$iff it intersects $v_{p}$. The parameter $\delta$ must be chosen sufficiently small so that in the vertical projection of the lines onto the $x y$-plane $v_{p}^{-}$and $v_{p}^{+}$are adjacent for all $p \in P$. For $\varepsilon>0$ we define $h_{l}^{-}$as the orthogonal projection of $l$ onto the plane $x=\sigma_{l}-\varepsilon$ and $h_{l}^{+}$as the orthogonal projection of $l$ onto $x=\sigma_{l}+\varepsilon\left(h_{l}^{-}\right.$and $h_{l}^{+}$are just translates of $h_{l}$ along the $x$-axis). If $h_{l}$ intersects $v_{p}$ then we get

$$
v_{p}^{-}<h_{l}^{+}<v_{p}^{+}<h_{l}^{-}<v_{p}^{-},
$$

a tetragonal cycle in the weaving defined by $H_{2}=\left\{h_{l}^{-}, h_{l}^{+} \mid h_{l} \in H_{1}\right\}$ and $V_{2}=$ $\left\{v_{p}^{-}, v_{p}^{+} \mid v_{p} \in V_{1}\right\}$, see Fig. 5 .

To get the final weaving we need to slightly modify the current collection of lines in space. First, we rotate the entire configuration of lines by an angle $\phi>0$ about the $y$-axis to avoid that lines $h_{l_{1}}$ and $h_{l_{2}}$ have the same vertical projection even if $l_{1}$ and $l_{2}$ have the same slope. This rotation is necessary because $L$ is such that many of its lines have the same slope. The angle $\phi$ must be chosen small enough so that the projections onto the $x y$-plane of any two lines in $H_{2}$ do not intersect inside a circle that encloses all intersections between projections of lines in $H_{2}$ and projections of lines in $V_{2}$. Depending on $\phi$, the parameter $\varepsilon$ has to be chosen small enough so that in the vertical projection onto the $x y$-plane $h_{l}^{-}$and

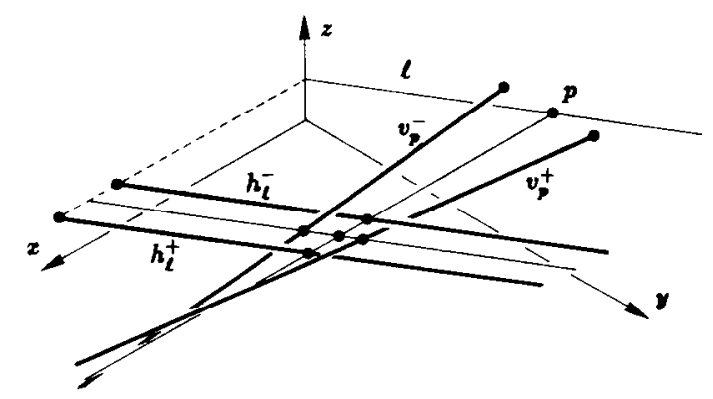

Fig. 5. Constructing an elementary cycle from a point-line incidence in the $y z$-plane. 
$h_{l}^{+}$are adjacent for all $l \in L$. This is possible because the choice of $\varepsilon$ does not affect the choice of $\phi$. Finally, we clip the lines outside the circle mentioned above and get two sets of rods, set $H$ from $H_{2}$ and $V$ from $V_{2}$. We have $|H|=|V|=n$, and the weaving defined by $H$ and $V$ has the desired number of elementary cycles.

Remarks. (1) It is not difficult to extend the bounds for elementary cycles to tetragonal cycles of fixed size. For integers $k, l \geqslant 1$ call a tetragonal cycle $h_{i}<v_{j}<h_{i+k}<v_{j+l}<h_{i}$ a $(k, l)$-cycle; so a $(1,1)$-cycle is an elementary cycle. Assuming $k \leqslant l$ we can obtain $\Omega\left(k^{1 / 3} l^{1 / 3} n^{4 / 3}+l n\right)$ as a lower bound and $\mathrm{O}\left(k^{1 / 2} n^{3 / 2}+\ln \right)$ as an upper bound for the maximum number of $(k, l)$-cycles.

(2) If we sum the upper bounds on the numbers of $(k, l)$-cycles for all $k$ and $l$ with $1 \leqslant k \leqslant l \leqslant m$ we get $\mathrm{O}\left(m^{5 / 2} n^{3 / 2}+m^{3} n\right)$. This is an upper bound on the maximum total number of $(k, l)$-cycles for $k, l \leqslant m$ since the maximum number of $(l, k)$-cycles is the same as the maximum number of $(k, l)$-cycles. In the next section we will remark that this suffices to show that $\mathrm{O}\left(n^{13 / 7}\right)$ cuts are enough to eliminate all cycles in a realizable $n$-by- $n$ weaving. Most likely, the upper bound on the total number of $(k, l)$-cycles for $k, l \leqslant m$ is not tight, but we will see how to decrease the number of cuts to $O\left(n^{y / 5}\right)$ without improving this upper bound.

\section{Cutting cycles in the bipartite case}

Let $\mathscr{W}$ be a realizable $n$-by- $n$ weaving defined by sets $H$ and $V$ of $n$ rods in space each. To cut a rod means to remove one of its points which, in effect, decomposes the rod into two rods. The goal of this section is to show that it is always possible to remove all cycles in a realizable bipartite weaving with relatively few cuts. Let $g(n)$ be the maximum, taken over all realizable $n$-by- $n$ weavings, of the minimum number of cuts needed to remove all cycles. Trivially, we have $\frac{1}{4} c_{1} n^{4 / 3} \leqslant g(n) \leqslant$ $n^{2}$, where the lower bound follows from the lower bound of Theorem 3.1, and the upper bound holds because it is certainly sufficient to cut every rod of one set where it crosses over or under the rods of the other set.

\subsection{A topological lemma}

Before discussing strategies that remove all cycles with a subquadratic number of cuts, let us convince ourselves that it suffices to cut tetragonal cycles. We first introduce some notation. For rods $h \in H$ and $v \in V$ we call $h^{\prime} \cap v^{\prime}$ their crossing point and, where convenient, we will use this term also for the points on $h$ and $v$ vertically above $h^{\prime} \cap v^{\prime}$. For a cycle of length $2 \lambda$,

$$
C: h_{i_{1}}<v_{j_{1}}<h_{i_{2}}<\cdots<h_{i_{\lambda}}<v_{j_{\lambda}}<h_{i_{1}} \text {, }
$$

we obtain a $2 \lambda$-gon $C^{\prime}$ (i.e. possibly self-intersecting polygonal closed curve with $2 \lambda$ edges) by drawing the piece of a line segment (the projection of a rod of the 

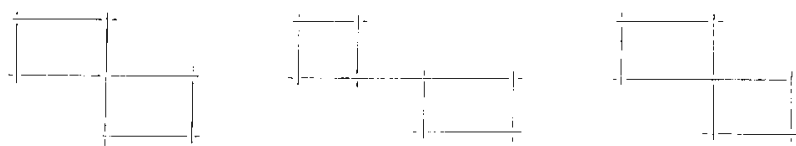

Fig. 6. To the left we see a cycle whose polygon has a bar and a post that properly intersect. In the middle the polygon has two bars that overlap along a line segment. The polygon to the right has a vertex common to two bars and to two posts.

cycle) between its crossing points with the preceding line segment and the succeeding line segment. We call $C^{\prime}$ the polygon of $C$ and augment each vertex and point of self-intersection with a bit that indicates which of the two rods crosses above the other at this point (see Figs. 6 and 7). For convenience we call the edges of $C^{\prime}$ that lie on line segments $h_{i}^{\prime}$ bars and we call the edges on line segments $v_{j}^{\prime}$ posts.

The following statement about cycles in a bipartite weaving is topological in the sense that it makes no reference to the realizability or nonrealizability of the weaving by rods.

Lemma 4.1. Any cycle $C$ in a bipartite weaving contains a tetragonal subcycle $T$ so that the bars of $T^{\prime}$ are (portions of) bars of $C^{\prime}$.

Proof. Let $C: h_{i_{1}}<v_{j_{1}}<h_{i_{2}}<\cdots<h_{i_{\lambda}}<v_{j_{\lambda}}<h_{i_{1}}$ be a nontetragonal cycle, that is, $\lambda>2$. We show below that $C$ has a proper subcycle $D$ with the property that all bars of $D^{\prime}$, the polygon of $D$, are (portions of) bars of $C^{\prime}$, the polygon of $C$. By repeated application of this argument we eventually get a tetragonal subcycle $T$ of $C$ so that the two bars of $T^{\prime}$ are (portions of) bars of $C^{\prime}$. We distinguish between the case when $C^{\prime}$ is self-intersecting and when it is a simple polygon.

Case 1: $C^{\prime}$ has a point of self-intersection $p$ (Fig. 6).

If $p$ lies in the intersection of two bars corresponding to the same rod $h=h_{i_{k}}=h_{i_{i}}$ with $k<l$ then

$$
h<v_{i_{k}}<h_{i_{k+1}}<\cdots<v_{j_{l-1}}<h
$$

is a proper subcycle of $C$ (Fig. 6 middle and right). Symmetrically, we find such a subcycle if $p$ belongs to two posts corresponding to a common rod. Otherwise, let $p$ be the crossing point of $h_{i_{k}}$ and $v_{j}$, and assume without loss of generality that
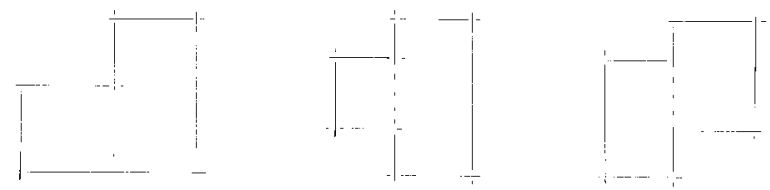

Fig. 7. To the left we have a post that hits a bar when it is extended. In the middle and to the right we see a rod in $V$ corresponding to two non-intersection posts which hit another reflex vertex when they are extended. 
$k<l$ (Fig. 6 left). If $h_{i_{k}}<v_{j_{l}}$ then

$$
h_{i_{1}}<\cdots<h_{i_{k}}<v_{j_{l}}<\cdots<v_{j_{h}}<h_{i_{1}}
$$

is a proper subcycle of $C$. Otherwise, i.e. if $v_{j_{l}}<h_{i_{k}}$, then

$$
h_{i_{k}}<\cdots<v_{j_{l}}<h_{i_{k}}
$$

is a proper subcycle of $C$. In all cases all edges of the polygon of the subcycle are edges or portions of edges of $C^{\prime}$.

Case 2: $C^{\prime}$ is a simple polygon.

Call a vertex of $C^{\prime}$ convex if its angle inside $C^{\prime}$ is less than $\pi$ and call it reflex if it is greater than $\pi$. The convex vertices of $C^{\prime}$ outnumber the reflex vertices by 4 . It follows that there are $\lambda-2>0$ reflex vertices. Let $q$ be a reflex vertex of $C^{\prime}$ and assume it is the crossing point of $h_{i_{k}}$ and $v_{i_{k}}$ (the case where $q$ is the crossing point of $h_{i_{k}}$ and $v_{j_{k-1}}$ is similar). Extend the post incident to $q$ inside $C^{\prime}$ until it hits another reflex vertex (Fig. 4 middle and right) or a bar of $C^{\prime}$ (Fig. 7 left). In the former case, let $v=v_{j_{k}}=v_{j_{t}}$ with $k<l$ be the rod corresponding to the two posts that become connected when one of them is extended. Then

$$
v<h_{i_{k+1}}<v_{i_{k+1}}<\cdots<h_{i_{t}}<v
$$

is a proper subcycle $D$ of $C$. In the latter case, define $l$ so that the bar hit lies on $h_{i_{l}}^{\prime}$ and assume $k<l$ without loss of generality. If $v_{j_{k}}<h_{i_{l}}$ then

$$
h_{i_{1}}<\cdots<v_{j_{k}}<h_{i_{l}}<\cdots<v_{j_{i}}<h_{i_{1}}
$$

is a proper subcycle $D$ of $C$. Otherwise,

$$
v_{j_{k}}<\cdots<h_{i_{l}}<v_{j_{k}}
$$

is such a $D$. In all cases, $D^{\prime}$ is part of $C^{\prime}$, except for the extension of the post. In particular, all bars of $D^{\prime}$ are (portions of) bars of $C^{\prime}$.

Lemma 4.1 implies that if we cut each tetragonal cycle of $\mathscr{W}$ at one of its bars then no cycle remains. By symmetry, the same is true if we cut every tetragonal cycle at one of its posts. Observe, however, that if we cut some tetragonal cycles at their bars and others at their posts, then there is no guarantee that the resulting set of rods is cycle-free.

Here is another topological observation about the cycle structure in a bipartite weaving that will be useful in bounding the number of cuts necessary to eliminate all cycles in a realizable bipartite weaving. Let

$$
C: h_{i_{1}}<v_{j_{1}}<h_{i_{2}}<v_{j_{3}}<h_{i_{1}} \text { and } D: h_{i_{1}}<v_{j_{2}}<h_{i_{2}}<v_{j_{4}}<h_{i_{1}}
$$

be two ccw tetragonal cycles with $i_{1}<i_{2}$ and $j_{1}<j_{2}<j_{3}<j_{4}$ (see Fig. 8). Then

$$
E: h_{i_{1}}<v_{j_{2}}<h_{i_{2}}<v_{j_{3}}<h_{i_{1}}
$$

is also a ccw tetragonal cycle. The symmetric statement holds for cw tetragonal cycles. Note that the region bounded by $E^{\prime}$ is contained in the regions of $C^{\prime}$ and 


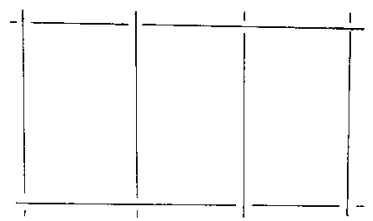

Fig. 8. If two tetragonal cycles with overlapping polygon regions share the rods in $H$ then there is another tetragonal cycle whose region is where the former two polygon regions overlap.

$D^{\prime}$ and that the bars of $E^{\prime}$ lie on the bars of $C^{\prime}$ and $D^{\prime}$. Thus, if we cut $E$ at its bars we automatically eliminate also $C$ and $D$.

\subsection{A subquadratic bound}

The strategy for eliminating all cycles in $\mathscr{W}$ consists of two steps. For the first step choose an integer $m$ and cut all rods of $V$ after every $m$ th rod of $H$ and all rods of $H$ after every $m$ th rod of $V$. After this step, any remaining cycle has all its rods contained within $m$ contiguous rods of $H$ and $m$ contiguous rods of $V$. In particular, all remaining tetragonal cycles are $(k, l)$-cycles with $k, l \leqslant m$. The second step cuts every remaining tetragonal cycle at one of its two bars; by Lemma 4.1 this eliminates all cycles.

As noted in Remark (2) after Theorem 3.1, there are only $O\left(m^{5 / 2} n^{3 / 2} \mid m^{3} n\right)$ tetragonal cycles left after the first step, so the same number of cuts is certainly sufficient. Since the first step makes $\mathrm{O}\left(n^{2} / m\right)$ cuts we are well advised to choose $m=\left|n^{1 / 7}\right|$ in which case the total number of cuts is $\mathrm{O}\left(n^{13 / 7}\right)$. We are now going to improve this bound using the observation about tetragonal cycles with overlapping regions.

Theorem 4.2. The number of cuts necessary to eliminate all cycles in a realizable bipartite $n$-by- $n$ weaving is $\mathrm{O}\left(n^{9 / 5}\right)$.

Proof. We choose $m=\left\lfloor n^{1 / 5}\right\rfloor$ and show that $\mathrm{O}\left(m^{3 / 2} n^{3 / 2}+m^{3} n\right)$ cuts suffice to eliminate all $(k, l)$-cycles, for $k, l \leqslant m$, with the additional property that each $(k, l)$-cycle is cut at one of its bars. To prove this claim we construct a bipartite graph whose nodes represent pairs of rods in $H$ and pairs in $V$ and whose arcs represent $\mathrm{ccw}$ tetragonal cycles that we choose to cut. This graph will contain many $K_{2,2}$ 's, but we will be able to decompose it into a rcasonably small number of graphs without $K_{2,2}$. The bound will then follow.

The bipartite graph $\mathscr{G}=(\bar{H} \cup \bar{V}, A)$ is defined as follows. $\bar{H}=\left\{\bar{h}_{i k}=\right.$ $\left.\left\{h_{i}, h_{i+k}\right\} \mid 1 \leqslant k \leqslant m, 1 \leqslant i \leqslant n-k\right\}, \bar{V}=\left\{\bar{v}_{j l}=\left\{v_{j}, v_{j+l}\right\} \mid 1 \leqslant l \leqslant m, 1 \leqslant j \leqslant n-\right.$ $l\}$, and $\left\{\bar{h}_{i k}, \bar{v}_{j l}\right\} \in A$ if:

(i) it defines a ccw cycle, that is, $h_{i}<v_{j}<h_{i+k}<v_{j+1}<h_{i}$, and

(ii) there are no two indices $\mu \geqslant j$ and $v<l$ with $\mu+v \leqslant j+l$ so that $\left\{\bar{h}_{i k}, \bar{v}_{\mu v}\right\}$ defines a ccw cycle. 
Trivially, we have $|\bar{H}|=|\bar{V}| \leqslant m n$. It should be clear that each arc in $A$ represents a ccw $(k, l)$-cycle, with $k, l \leqslant m$, and that is is sufficient to cut each represented cycle at one of its bars. Together with the cuts of the first step these cuts will eliminate all ccw cycles. The cw cycles can be taken care of by a symmetric argument.

It remains to show that $|A|=\mathrm{O}\left(n^{9 / 5}\right)$. To this end we partition $\bar{H}$ into $\left(\begin{array}{c}m+1 \\ 2\end{array}\right)$ sets $\bar{H}_{a b}$, for integers $1 \leqslant a \leqslant b \leqslant m$, and for each set $\bar{H}_{a b}$ we define $\mathscr{G}_{a b}=$ $\left(\bar{H}_{a b} \cup \bar{V}, A_{a b}\right)$ as the subgraph of $\mathscr{G}$ induced by $\bar{H}_{a b} \cup \bar{V}$. Clearly, we have $A=\bigcup_{1 \leqslant a \leqslant b \leqslant m} A_{a b}$. An important property of the partition will be that each graph $\mathscr{G}_{a b}$ has no $K_{2,2}$. From this property and the extremal graph lemma mentioned earlier it follows immediately that

$$
\begin{aligned}
|A| & =\sum_{1 \leqslant a \leqslant b \leqslant m}\left|A_{a b}\right| \\
& \leqslant c\left(\left(\sum_{1 \leqslant a \leqslant b \leqslant m}\left|\bar{H}_{a b}\right|\right) \sqrt{|\bar{V}|}+\left(\begin{array}{c}
m+1 \\
2
\end{array}\right)|\bar{V}|\right) \leqslant c\left(m^{3 / 2} n^{3 / 2}+m^{2} n\right)
\end{aligned}
$$

as claimed.

We now finish the proof by specifying the sets $\bar{H}_{a b}$ that satisfy the requirements. Intuitively, $b$ is the difference between the indices of the two rods of the pairs in $\bar{H}_{a b}$ and $a$ is the index modulo $b$ of the first rod. Formally, $\bar{H}_{a b}=$ $\left.\left\{h_{i}, h_{i+b}\right\} \mid a=i(\bmod b)\right\}$. We have to show that $\mathscr{G}_{a b}$ contains no $K_{2,2}$. Assume it does and let $\bar{h}_{i_{1} i_{2}}, \bar{h}_{i_{3_{i}} i_{4}}, \bar{v}_{j_{1} j_{2}}$, and $\bar{v}_{j_{3} j_{4}}$ be the four nodes of a $K_{2,2}$. By definition of $\bar{H}_{a b}$ we have $i_{1}<i_{1}+i_{2} \leqslant i_{3}<i_{3}+i_{4}$, assuming $i_{1}<i_{3}$; moreover, $i_{1}+i_{2}$ cannot be equal to $i_{3}$ because both $\left\{\bar{h}_{i_{1} i_{2}}, \bar{v}_{j_{1} j_{2}}\right\}$ and $\left\{\bar{h}_{i_{3} i_{4}}, \bar{v}_{j_{1} j_{2}}\right\}$ define a ccw cycle. Furthermore, $j_{1} \neq j_{3}$ because otherwise the region bounded by the polygon of $\left\{\bar{h}_{i_{1} i_{2}}, \bar{v}_{j_{1} j_{2}}\right\}$ is contained in the region of $\left\{\bar{h}_{i_{1} i_{2}}, \bar{v}_{j_{3} j_{4}}\right\}$ or vice versa, which is a contradiction to condition (ii) of the definition of $\mathscr{G}$. So assume $j_{1}<j_{3}$. We have $j_{1}<j_{1}+j_{2} \leqslant j_{3}<j_{3}+j_{4}$, and $j_{1}+j_{2} \neq j_{3}$ because the cycles defined by $\left\{\bar{h}_{i_{1} i_{2}}, \bar{v}_{i_{1} j_{2}}\right\}$ and $\left\{\bar{h}_{i_{1} i_{2}}, \bar{v}_{j_{3} j_{4}}\right\}$ are both ccw. Thus, we have $i_{1}<i_{1}+i_{2}<i_{3}<i_{3}+i_{4}$ and $j_{1}<j_{1}+j_{2}<j_{3}<j_{3}+j_{4}$. But if the four cycles defined by $\left\{\bar{h}_{i_{1} i_{2}}, \bar{v}_{j_{1} j_{2}}\right\},\left\{\bar{h}_{i_{1} i_{2}}, \bar{v}_{j_{3} j_{4}}\right\}$, $\left\{\bar{h}_{i_{3} i_{4}}, \bar{v}_{j_{1} j_{2}}\right\}$, and $\left\{\bar{h}_{i_{3 i_{4}}}, \bar{v}_{j_{3} j_{4}}\right\}$ are all ccw then the eight rods form a perfect 4-by-4 weaving, which is not realizable.

\section{A combinatorial incidence problem}

In an attempt to understand the combinatorics of cycles in the general, i.e. not necessarily bipartite case, we study the following combinatorial incidence problem. Call a point that is the intersection of three non-coplanar lines a joint. What is the maximum number of joints defined by a set of $n$ lines in space? The rationale behind studying joints is that a local change permits us to open a joint into an elementary cycle. Incidentally, this is precisely the connection between the lower bound on the number of joints (next paragraph) and the lower bound on the number of elementary cycles (Section 6). 
It is fairly easy to prove that $\Omega\left(n^{3 / 2}\right)$ is a lower bound for the maximum number of joints. Take $k$ planes in general position and observe that they intersect pairwise in $n=\left(\begin{array}{l}k \\ 2\end{array}\right)$ lines. We have $\left(\begin{array}{l}k \\ 3\end{array}\right)-\Omega\left(n^{3 / 2}\right)$ joints at the intersections of any three planes.

The main result of this section is a subquadratic upper bound on the number of joints defined by $n$ lines. Its proof is self-contained, except for a result on extremal graphs and some properties of quadratic surfaces. The specific result on graphs we use is that a graph with $n$ nodes and no $K_{3, t}$ has at most $\mathrm{O}\left(t^{1 / 3} n^{5 / 3}\right)$ arcs (see e.g. [1, chapter VI]).

Among the quadratic surfaces (quadrics) in space we are interested in those that contain lines. Aside from cylinders and cones there are two kinds of such surfaces (called reguli), namely hyperboloids of one sheet and hyperbolic paraboloids (see e.g. [15]). Being a quadratic surface, a regulus intersects any line in at most two points or it contains the entire line. Another property we use is that any regulus $q$ is ruled by two disjoint infinite families of lines, $L_{1}$ and $L_{2}$, that is, $q=\bigcup_{l \in L_{1}} l=\bigcup_{l \in L_{2}} l$. Call two lines skew if they are disjoint and not parallel. The lines in $L_{1}$ and $L_{2}$ have the property that any pair from $L_{1}$ or from $L_{2}$ is skew and every line in $L_{1}$ mects or is parallel to every line in $L_{2}$. Furthermore, for any three pairwise skew lines there is a unique regulus that contains the three lines. We are now ready to prove the upper bound.

Theorem 5.1. The number of joints defined by a set of $n$ lines in space is $\mathrm{O}\left(n^{7 / 4}\right)$.

Proof. Let $L$ be a set of $n$ lines in space, and let $\mathscr{G}=(N, A)$ be its intersection graph, that is, $N=L$ and $\left\{l_{1}, l_{2}\right\} \in A$ iff $l_{1} \cap l_{2} \neq \emptyset$. Clearly, $|A|$ is an upper bound on the number of joints defined by $L$. However, at this stage $\mathscr{G}$ could be the complete graph and it will take some pruning to get a subquadratic upper bound on the number of joints using $\mathscr{G}$. The pruning is done in three steps: first, we remove joints of high degree, second, we remove planes containing many lines, and third, we remove reguli containing many lines. Let $\tau$ be a positive integer to be specified later.

Step 1. For each joint $p$ incident to $i(p) \geqslant \tau$ lines in $L$ we remove from $A$ the $\left(\begin{array}{c}i(p) \\ 2\end{array}\right)$ arcs $\left\{l_{1}, l_{2}\right\}$ with $l_{1} \cap l_{2}=p$. After removing these arcs, $p$ is an orphan, that is, there is no arc left witnessing that $p$ is a joint. At the end of Step 1, the number of orphan joints is at most $n(n-1) / \tau(\tau-1)$.

Step 2. As long as there is a plane $h$ containing $j(h) \geqslant \tau$ lines of $N$ we remove from $N$ the $j(h)$ lines and from $A$ the incident arcs. Each line not contained in $h$ meets $h$ in at most one point, which implies that $h$ contains at most $n-j(h) \leqslant n$ joints and thus its deletion generates at most the same number of new orphans. The number of planes $h$ considered is at most $n / \tau$, which implies that Step 2 adds at most $n^{2} / \tau$ orphans to those created in Step 1 .

Step 3. As long as there is as regulus $q$ so that $j(q) \geqslant \tau$ lines lie on $q$ we remove from $N$ the $j(q)$ lines and from $A$ the incident arcs. Each line not contained in $q$ 
meets $q$ in at most two points, which implies that $q$ contains at most $2 n-2 j(q) \leqslant 2 n$ joints. The number of reguli considered is at most $n / \tau$. Hence, Step 3 turns at most $2 n^{2} / \tau$ joints to orphans.

We argue that after the three steps $\mathscr{G}$ contains no $K_{3,2 \tau}$. Suppose $\mathscr{G}$ contains a $K_{3,2 \tau}$ and let the lines that form this subgraph be $a_{1}, a_{2}, a_{3}$ and $b_{1}, b_{2}, \ldots, b_{2 x}$. Assume first that $a_{1}$ and $a_{2}$ are not skew. If they are parallel then all lines $b_{i}$ lie on the plane defined by $a_{1}$ and $a_{2}$, contradicting Step 2. If $a_{1} \cap a_{2}=p$ then at least half of the $b_{i}$ go through $p$, contradicting Step 1, or at least half lie in the plane defined by $a_{1}$ and $a_{2}$, contradicting Step 2. Finally, assume that $a_{1}, a_{2}, a_{3}$ are pairwise skew. Then they define a unique regulus with $a_{1}, a_{2}, a_{3}$ in one family of ruling lines and the $b_{i}$ in the other family, thus contradicting Step 3 .

After the three steps $\mathscr{G}$ contains no $K_{3,2 \tau}$ and therefore $|A|=\mathrm{O}\left(\tau^{1 / 3} n^{5 / 3}\right)$. This number is also an upper bound on the number of non-orphan joints-the number of orphan joints is $\mathrm{O}\left(n^{2} / \tau\right)$. The claim thus follows if we set $\tau=\left\lfloor n^{1 / 4}\right\rfloor$.

Remarks. (1) The problem of joints, has a dual version which is obtained by mapping points to polar planes, planes to polar points, and lines to polar lines. Call three lines concurrent if they meet in a common point or are parallel, and for a given set of lines call a plane a joining plane if it contains at least three nonconcurrent lines of the set. The dual version of Theorem 5.1 states that a set of $n$ lines in space defines at most $\mathrm{O}\left(n^{7 / 4}\right)$ joining planes.

(2) For the case where the set of lines can be partitioned into three classes of parallel lines, the results in [14] imply an upper bound of $\mathrm{O}\left(n^{3 / 2}\right)$ for the number of joints. This bound is tight.

\section{Counting elementary cycles in the general case}

In this section we consider realizable weavings that are not necessarily bipartite and study the maximum number of elementary cycles. To define the notion of an elementary cycle we need a few definitions.

Let $L$ be a set of $n$ rods in space and define $L^{\prime}=\left\{l^{\prime} \mid l \in L\right\}$, where $l^{\prime}$ is the vertical projection of $l$ onto the $x y$-plane. By the nature of the problem we may make the simplifying assumptions that each rod in $L$ is actually a line, that each $l^{\prime}$ is a line in the $x y$-plane (as opposed to a point), that no two lines in $L$ intersect, that any two lines in $L^{\prime}$ meet in a point, and that no three lines in $L^{\prime}$ meet in a common point. The lines in $L^{\prime}$ define a subdivision of the $x y$-plane known as the arrangement of $L^{\prime}$ [6]. It consists of $\left(\begin{array}{l}n \\ 2\end{array}\right)+n+1$ regions $\left(\begin{array}{c}n \\ 2\end{array}\right)-n+1$ of which are bounded. A cycle

$$
l_{1}<l_{2}<\cdots<l_{k}<l_{1}
$$

of lines in $L$ is elementary if there is a bounded region in the arrangement of $L^{\prime}$ 
whose boundary consists of $k$ edges supported by $l_{1}^{\prime}, l_{2}^{\prime}, \ldots, l_{k}^{\prime}$. We remark that an elementary cycle is not necessarily a shortest cycle in the sense that $k$ is minimal. In fact, our assumptions about $L$ imply that the digraph $\mathscr{G}=(L, A)$ with $\left(l_{i}, l_{j}\right) \in A$ iff $l_{i}<l_{j}$ is a tournament which implies that every cycle, and therefore also every elementary cycle, contains a shorter subcycle unless its length is 3 .

Since there are only $\left(\begin{array}{l}n \\ 2\end{array}\right)-n+1$ bounded regions in the arrangement of $L^{\prime}$ the number of elementary cycles in the weaving $\mathscr{W}$ defined by $L$ cannot exceed this number. If the number of elementary cycles were equal to the number of bounded regions then $\mathscr{W}$ would be perfect, that is, each line would strictly alternate between crossing 'over' and crossing 'under', see Fig. 9. As proved in [12], a perfect weaving of $n \geqslant 4$ lines is not realizable. This implies that the number of elementary cycles is strictly less than the number of bounded regions of the arrangement, provided $n \geqslant 4$. However, at this time no subquadratic upper bound on the number of elementary cycles is known, although Theorem 5.1 suggests that the number is subquadratic in $n$, maybe even $\mathrm{O}\left(n^{7 / 4}\right)$.

Observe that if we could prove that any realizable weaving of $n$ lines in space has a line that alternates at most a sublinear number of times between 'over' and 'under', then induction would establish a subquadratic upper bound on the number of elementary cycles. However, such a line does not necessarily exist, as is indicated by the realizable weaving on the right hand side of Fig. 9.

In the remainder of this section we show that $\Omega\left(n^{3 / 2}\right)$ is a lower bound for the maximum number of elementary cycles in a realizable weaving of $n$ lines. For the construction take $k$ planes in general position in space and consider the set $L$ of $n=\left(\begin{array}{l}k \\ 2\end{array}\right)$ lines of intersection. Let $\varepsilon>0$ be a sufficiently small real number. For a line $l \in L$ let $h_{+}$and $h_{-}$be the two planes that intersect in $l$. We define two lines $l_{+}$and $l_{-}$parallel to $l$, so that $l_{+}$lies in $h_{+}$and vertically above $h_{-}, l_{-}$lies in $h_{-}$ and vertically above $h_{+}$, and $l_{+}^{\prime}$ and $l_{-}^{\prime}$ each lie at distance $\varepsilon$ from $l^{\prime}$ ( Fig. 10).

We form a set $L_{*}=\left\{l_{*} \mid l \in L\right\}$ where $l_{*}$ is randomly chosen to be either $l_{+}$or $l_{-}$. Let $p$ be the point of intersection of three lines $l_{1}^{\prime}, l_{2}^{\prime}, l_{3}^{\prime} \in L^{\prime}$ and consider the hexagon defined by $l_{1+}^{\prime}, l_{1-}^{\prime}, l_{2+}^{\prime}, l_{2-}^{\prime}, l_{3+}^{\prime}, l_{3-}^{\prime}$. If $l_{1_{*}}^{\prime}, l_{2 *}^{\prime}, l_{3 *}^{\prime}$ contain three pairwise non-adjacent edges of the hexagon then they form an elementary cycle.
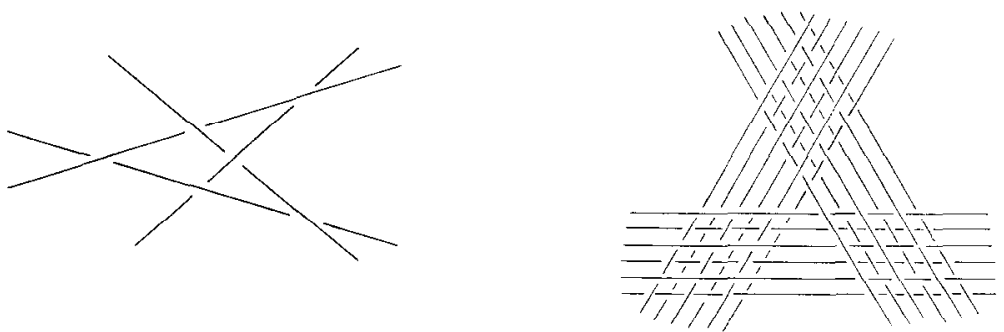

Fig. 9. The weaving to the left is perfect but not realizable; each bounded region corresponds to an elementary cycle. In the weaving to the right, which is realizable, each line alternates at least about $n / 3$ times between 'over' and 'under', still there is not a single elementary cycle. 


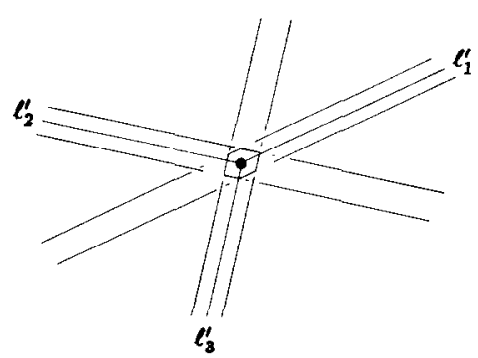

Fig. 10. If $l_{1}^{\prime}, l_{2}^{\prime}, l_{3}^{\prime} \in L^{\prime}$ meet in a common point $p$ then $l_{1+}^{\prime}, l_{1-}^{\prime}, l_{2+}^{\prime}, l_{2-}^{\prime}, l_{3+}^{\prime}, l_{3-}^{\prime}$ form a hexagonal region around $p$.

since they are $2^{3}=8$ ways to choose the lines $l_{1 *}, l_{2 *}, l_{3 *}$ and two choices lead to an elementary cycle we conclude that the expected number of elementary cycles in the weaving defined by $L_{*}$ is at least $\frac{2}{8}\left(\begin{array}{l}k \\ 3\end{array}\right)=\Omega\left(n^{3 / 2}\right)$. Therefore, there is a choice of $L_{*}$ with at least this many elementary cycles.

Remark. One aspect of the above construction is its close relationship to the problem of joints of the previous section.

\section{Discussion}

This paper presents several algorithmic and combinatorial results for problems about cycles in a view of a finite set of rods and lines in threcdimensional space. The problems considered are motivated by hidden surface removal algorithms, such as the painter's algorithm, and include sorting the rods or lines, bounding the number of cycles they can form, and cutting cycles. Our investigations leave and raise a number of open problems including the general quest to improve the bounds obtained in Theorems 2.1, 3.1, 4.2, and 5.1. We also state a few more specific problems.

(1) Prove a subquadratic upper bound on the number of elementary cycles in a realizable weaving defined by $n$ lines in space. The current best lower bound is $\Omega\left(n^{3 / 2}\right)$ as demonstrated in Section 6.

(2) Show that a subquadratic number of cuts is sufficient to eliminate all cycles in a view of a set of $n$ rods or lines in space. The lower bound on the number of elementary cycles implies that $\Omega\left(n^{3 / 2}\right)$ cuts are sometimes necessary. A related problem is to cut the rods or lines so that all views of the set are free of cycles. Paterson and Yao [13] show that $\mathrm{O}\left(n^{2}\right)$ cuts are sufficient for this more general problem. Is this bound tight or is a subquadratic number of cuts sufficient?

(3) In Section 4 we show that $\mathrm{O}\left(n^{9 / 5}\right)$ cuts are sufficient to eliminate all cycles in a realizable bipartite $n$-by- $n$ weaving, but we have no fast algorithm that finds these cuts. 
(4) Another interesting algorithmic question is to sort $n$ rods in space in subquadratic time, assuming the rods form no cycle (see also the remark after Theorem 2.1).

\section{Acknowledgements}

The authors express their thanks for the generous support of the DEC Systems Research Center at Palo Alto where a good portion of the reported research was performed

\section{References}

[1] B. Bollobás, Extremal Graph Theory (Academic Press, London, 1978).

[2] B. Chazelle and H. Edelsbrunner, An optimal algorithm for intersecting line segments in the plane, J. Assoc. Comput. Mach. 39 (1992) 1-54.

[3] B. Chazelle, H. Edelsbrunner, L.J. Guibas and M. Sharir, Algorithms for bichromatic line segment problems and polyhedral terrains, Rept. UIUCDCS-R-90-1578, Dept. Comput. Sci., Univ. Illinois, Urbana, Illinois, 1990.

[4] B. Chazelle, H. Edelsbrunner, L.J. Guibas, M. Sharir and J. Stolfi, Lines in space: combinatorics and algorithms, Rept. UIUCDCS-R-90-1569, Dept. Comput. Sci., Univ. Illinois, Urbana, Illinois, 1990.

[5] K.L. Clarkson, H. Edelsbrunner, L.J. Guibas, M. Sharir and E. Welzl, Combinatorial complexity bounds for arrangements of curves and spheres, Discrete Comput. Geom. 5 (1990) 99-160.

[6] H. Edelsbrunner, Algorithms in Combinatorial Geometry (Springer, Berlin, 1987).

[7] J.D. Foley and A. van Dam, Fundamentals of Interactive Computer Graphics (Addison-Wesley, Reading, MA, 1982).

[8] H. Fuchs, Z. Kedem and B. Naylor, On visible surface generation by a priori tree structures, Computer Graphics (SIGGRAPG ' 80 conference proccedings) 124-133.

[9] K. Melhorn, Data Structures and Algorithms 1; Sorting and Searching (Springer, Berlin, 1984).

[10] K. Mehlhorn, Data Structures and Algorithms 2: Graph Algorithms and NP-Completeness (Springer, Berlin, 1984).

[11] M.H. Overmars and M. Sharir, Output-sensitive hidden surface removal, in: Proc. 30th Ann. IEEE Sympos. Found. Comput. Sci. 1989, 598-603.

[12] J. Pach, R. Pollack and E. Welzl, Weaving patterns of lines and line segments in space, in: Proc. Internat. Sympos. on Algorithms, SIGAL '90, Lecture Notes in Comput. Sci. 450 (Springer, Berlin, 1990) 439-446.

[13] M.S. Paterson and F.F. Yao, Binary partitions with applications to hidden-surface removal and solid modelling, in: Proc. 5th Ann. Sympos. Comput. Geom. 1989, $23-32$.

[14] M.S. Paterson and F.F. Yao, Optimal hinary space partitions for orthogonal objects, in: Proc. 1st ACM-SIAM Sympos. Discrete Algorithms, 1990, 100-106, to appear.

[15] D.M.Y. Sommerville, Analytic Geometry of Three Dimensions (Cambridge Univ. Press, Cambridge, 1934).

[16] E. Szemerédi and W.T. Trotter Jr., Extremal problems in discrete geometry, Combinatorica 3 (1983) 381-392.

[17] O. Ya. Viro, Topological problems concerning lines and points of three-dimensional space, Soviet Math. Dokl. 32 (1985) 528-531.

[18] W. Whiteley, Rigidity and polarity II: weaving lines and tensegrity frameworks, Geom. Dedicata 30 (1989) 255-279. 